\title{
The Ultraviolet View of the Magellanic Clouds from GALEX: A First Look at the LMC Source Catalog
}

\author{
Raymond Simons ${ }^{\mathrm{a}, *}$, David Thilker ${ }^{\mathrm{a}, * *}$, Luciana Bianchi ${ }^{\mathrm{a}}$, Ted Wyder ${ }^{\mathrm{b}}$ \\ ${ }^{a}$ Department of Physics and Astronomy, Johns Hopkins University, 3400 N. Charles Street, Baltimore, MD \\ 21218, USA \\ ${ }^{b}$ Astronomy Department, Caltech, MC 249-17, 1200 E. California Blvd., Pasadena, CA 91125, USA
}

\begin{abstract}
The Galaxy Evolution Exporer (GALEX) has performed unprecedented imaging surveys of the Magellanic Clouds (MC) and their surrounding areas including the Magellanic

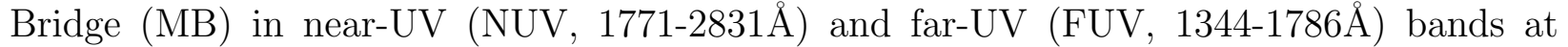
$5^{\prime \prime}$ resolution. Substantially more area was covered in the NUV than FUV, particularly in the bright central regions, because of the GALEX FUV detector failure. The $5 \sigma$ depth of the NUV imaging varies between 20.8 and 22.7 (ABmag). Such imaging provides the first sensitive view of the entire content of hot stars in the Magellanic System, revealing the presence of young populations even in sites with extremely low star-formation rate surface density like the MB, owing to high sensitivity of the UV data to hot stars and the dark sky at these wavelengths.

The density of UV sources is quite high in many areas of the LMC and SMC. Crowding limits the quality of source detection and photometry from the standard mission pipeline processing. We performed custom-photometry of the GALEX data in the MC survey region $\left(<15^{\circ}\right.$ from the $\mathrm{LMC},<10^{\circ}$ from the SMC). After merging multiple detections of sources in overlapping images, the resulting catalog we have produced for the LMC contains nearly 6 million unique NUV point sources within $15^{\circ}$ and is briefly presented herein. This paper provides a first look at the GALEX MC survey and highlights some of the science investigations that the entire catalog and imaging dataset will make possible.
\end{abstract}

Keywords: Ultraviolet: surveys; Astronomical Data Bases: catalogs; Galaxies: Magellanic Clouds; Ultraviolet: galaxies;

\section{Introduction}

As the nearest galaxies to the Milky Way, the Magellanic Clouds (MCs) have always been targets of considerable interest. The low metallicity (Olsen et al. 2011, Cole et al. 2005) of the Clouds, combined with their proximity $\left(D_{L M C}=50 \mathrm{kpc}\right.$ [Pietrzyński et al. 2013], $D_{S M C}$ $=61 \mathrm{kpc}$ [Hilditch et al. 2005]), allows for detailed observations and stellar evolution studies probing conditions rather different than in the Milky Way. However, comprehensive surveys

\footnotetext{
*rsimons@pha.jhu.edu

** Catalog correspondence to David Thilker, dthilker@pha.jhu.edu URL: http://dolomiti.pha.jhu.edu (Luciana Bianchi)
} 
become demanding and can only be efficiently performed with wide-field instruments. Large surveys of the Magellanic Clouds have been accomplished in optical and infrared bands, but a complete catalog of point sources in the ultraviolet (UV) has long remained missing due to the difficulty of obtaining the required observations from space.

The Magellanic Clouds have been previously observed in the UV by imaging and spectroscopic instruments ranging from rocket-borne cameras to HST, with a great range in resolution and areal coverage. Page \& Carruthers (1981) first observed the LMC in the wavelength range $1050-1600 \AA$ during the Apollo 16 mission, but at very low $\left(3^{\prime}\right)$ resolution. These Apollo observations delineated the overall UV morphology of the LMC very well and motivated continued efforts. This pathfinder study was followed by Smith, Cornett, \& Hill (1987) who imaged the entirety of the LMC in FUV and NUV bands at 50" resolution, still too low to resolve individual stars. Until GALEX, this work remained the highest quality panoramic imaging for either of the MCs. UIT, flown on the Space Shuttle during the Astro-1 and Astro-2 missions, also targeted the Clouds. These observations (LMC: Parker et al. 1998, 2001; SMC: Cornett et al. 1994, 1997; selected regions: Cheng et al. 1992, Hill et al. 1995) were of much higher resolution ( $\left.3^{\prime \prime}\right)$, even surpassing GALEX data in that respect. However, only a limited number of fields could be obtained during the Astro1+2 missions. FUSE, IUE and HST have provided numerous spectroscopic and small field-of-view imaging studies of the MCs (e.g. Brosch et al. 1999, Pradhan et al. 2011). At the time GALEX was launched, there remained a significant window for improvement in coverage and sensitivity over the best existing wide-field UV imaging (from UIT). The Science Team was keenly aware of this but had to be conservative in terms of brightness limits for the GALEX detectors, hence delaying the bulk of MC observations until the end of the NASA supported GALEX mission.

The GALEX mission (Martin et al. 2005, Morrissey et al. 2007, Bianchi 2009, 2011) has provided wide-field imaging in two UV bands, far-UV (FUV, 1344-1786 ) and near-UV $(\mathrm{NUV}, 1771-2831 \AA)$, with a field of view of $\approx 1.2^{\circ}$ diameter and a resolution of $\approx 4.2 / 5.3^{\prime \prime}(\mathrm{FUV} / \mathrm{NUV})$. In this paper, we present a first look from a comprehensive GALEX survey of the Magellanic Clouds and their environment, particularly focusing on the LMC UV source catalog while briefly outlining other planned studies.

\section{Data and Coverage}

For the study of the Magellanic System we include observations from all GALEX surveys (AIS, MIS, NGS, GI, etc., Martin et al. 2005) 1 for which the planned field center was either within $15^{\circ}$ of the LMC or $10^{\circ}$ from the SMC. These radial limits encompass a contiguous area of the sky including both Clouds, the entire Magellanic Bridge (MB), and some of the Magellanic Stream nearest to the galaxies (Fig. 1). A 'tile' defines the intended pointing of a GALEX observation, which may be performed with separate exposures (visits). All exposures of the same field are later coadded to improve S/N. We performed photometry on each visit-level image, rather than on coadds of repeated visits to the same tile. This allows us to track variability of sources if present, for those areas of the sky observed more

\footnotetext{
${ }^{1}$ AIS: All-Sky Imaging (100 s), MIS: Medium Imaging (1,500 s), NGS: Nearby Galaxy (1,500 s), GI: Guest Investigator
} 


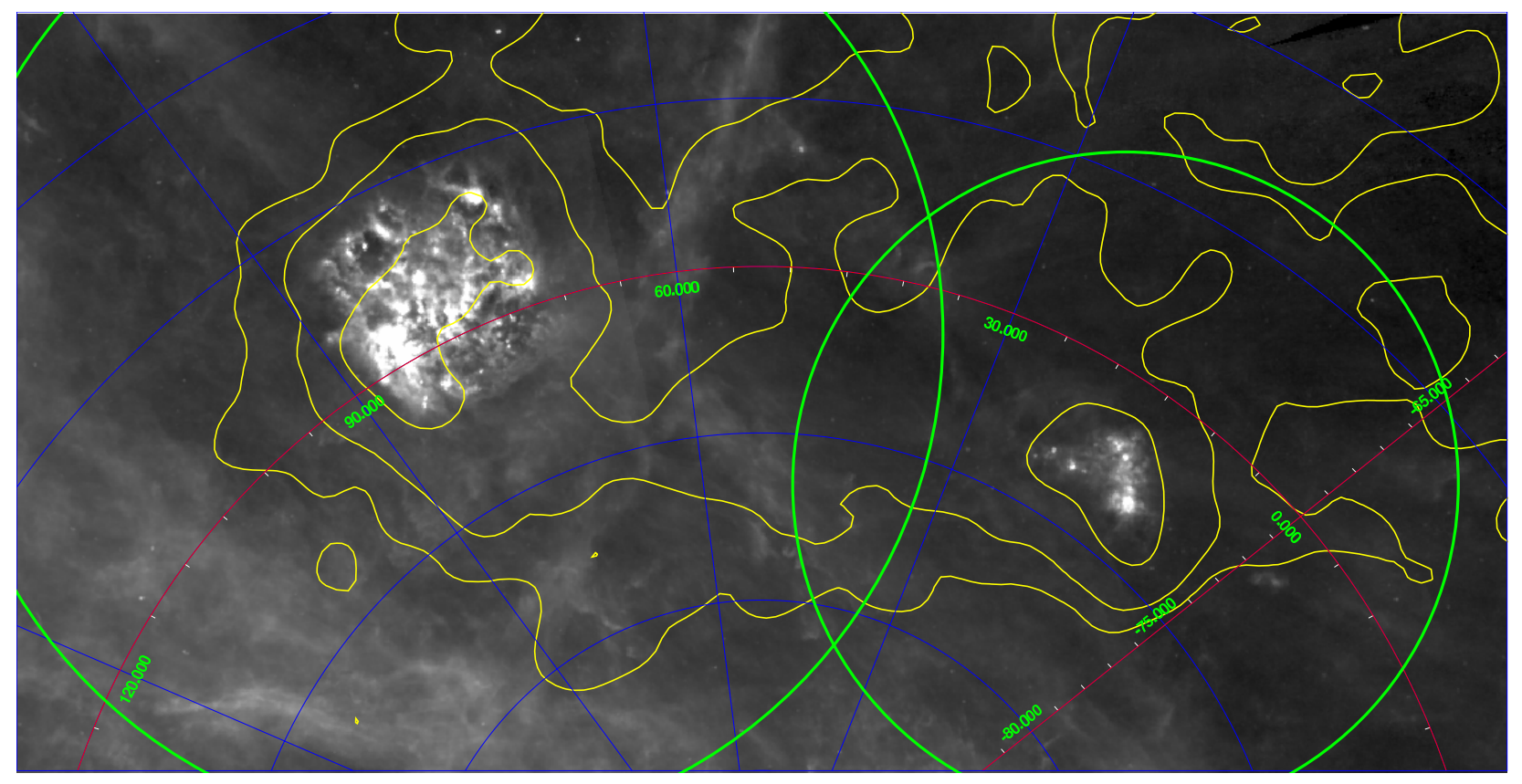

Figure 1: The GALEX Magellanic Cloud survey region as seen by IRAS at $100 \mu m$, contours of the HI distribution of Putman et al. (1998). The $\mathrm{N}(\mathrm{HI})$ contours are drawn at $10^{19}, 10^{20}$ and $10^{21} \mathrm{~cm}^{-2}$. We indicate the radial limits of our MC-specific GALEX analysis with the large circles having radius of $15^{\circ}$ and $10^{\circ}$, respectively for the LMC and SMC. Note that these limits include the entire Magellanic Bridge.

than once. Future work will fold in analysis of coadded data in order to push our catalogs to lower flux limits in selected regions.

The survey data have a range of exposure times. We have separated the overall dataset into two parts: observations which were part of the AIS (median exposure time of $~ 150$ s, 865 visits typically avoiding the bright areas of the Clouds, by design) and those which were not (384[294] visits more centrally concentrated on the LMC[SMC]). The distribution of visit NUV exposure times is presented in Fig. 2 for the LMC and SMC separately, along with a map of the accumulated total exposure across the survey area.

In our catalog result sections (Secs. 4 and 5), as a preview of forthcoming products, we only discuss the non-AIS subset pertaining to the LMC, having a median NUV exposure time of 733s. This first subset and source catalog presentation illustrates the type of data and the reduction (Sec. 3) which will apply to the complete survey. Full analysis of the entire Magellanic System catalog (all areas, all exposure depths) will be published subsequently elsewhere.

The image data were processed with the GALEX pipeline, and taken directly from the mission visit server at Caltech (internal access for the Science Team) since they are not yet completely in the MAST (galex.stsci.edu) public archives. The data will be identical when they are ingested in MAST. Some GALEX imaging presented here includes NUV-only data. This is because early observations of the MCs frequently encountered count-rate bright limit safety violations with consequent detector shutdowns in the FUV, and the FUV detector had already failed by the time subsequent attempts were acquired with relaxed safety thresholds. 

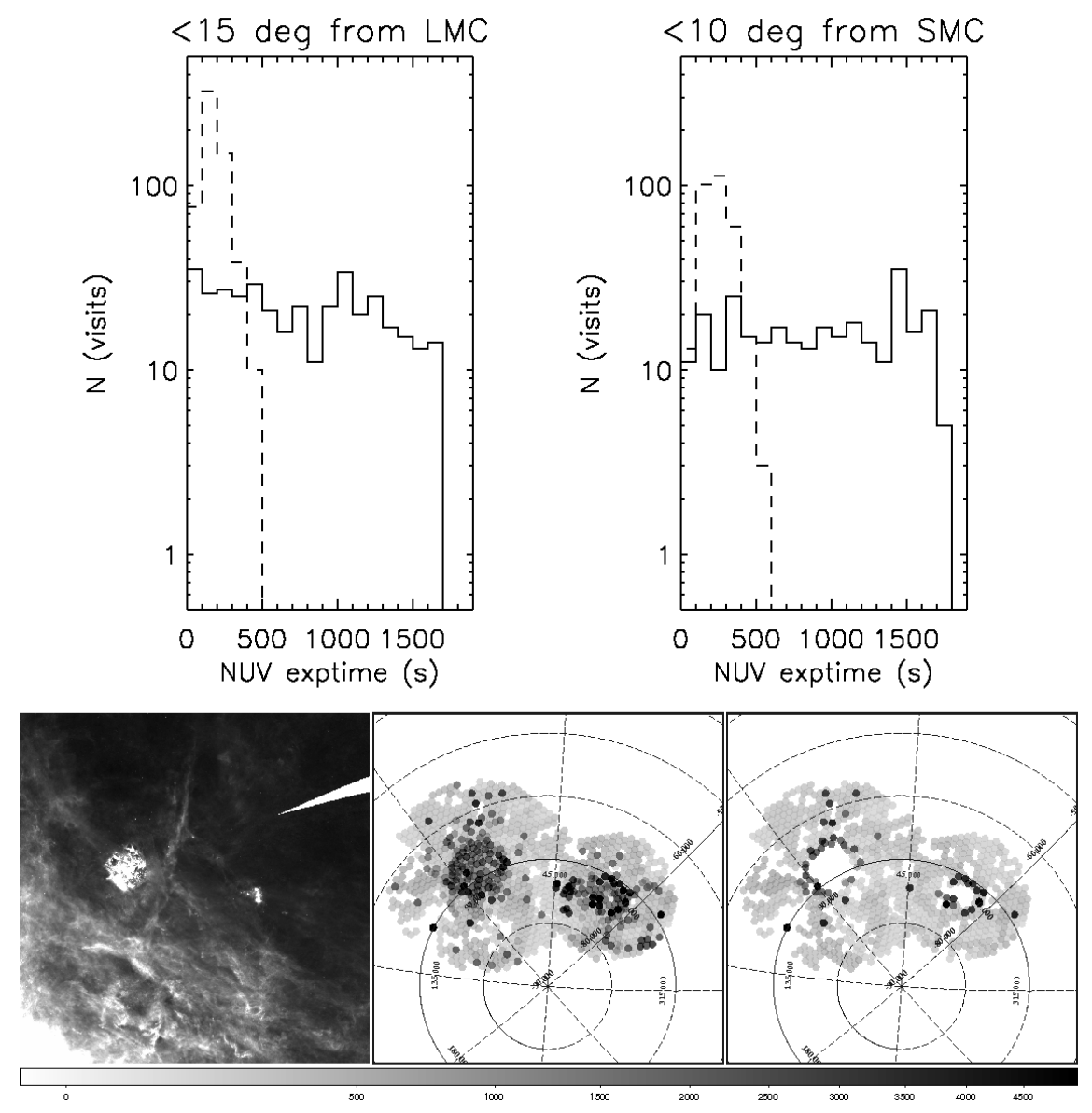

Figure 2: Distribution of GALEX exposure times in the NUV-band for non-AIS (solid line) and AIS (dashed line) visits falling within $15^{\circ}$ of the LMC (top left) or within $10^{\circ}$ of the SMC (top right). In the image panel, we display the total accumulated exposure time as a function of position for the NUV (bottom center) and FUV (bottom right), in comparison to the IRAS $100 \mu$ m survey data (bottom left) over a $55^{\circ}$-wide field.

The subset of visits with FUV data are a valuable resource for characterizing the nature of the sources and inferring their $\mathrm{T}_{\text {eff }}$ from FUV-NUV color (Section 6.4) even if they are largely confined to the periphery of the Clouds and MB.

\section{Post-pipeline GALEX Photometry}

In this section we describe the custom photometry processing steps applied to our GALEX dataset. As we will show, the GALEX pipeline measurements are not well-suited to environments as complex as the Magellanic Clouds.

\subsection{Source Detection}

The GALEX pipeline sometimes fails to resolve closely neighboring point sources in crowded fields (Figure 3). Often, such partially blended sources are identified as elongated single sources by the SExtractor-based pipeline, which was not designed for the level of 
crowding encountered in the MCs, even in the peripheral fields. A more sophisticated approach had to be used in dense fields with close and overlapping sources. A NUV-selected source list was generated using the DAOfind function (implemented in IDL) with a peak amplitude threshold proportional to the (exptime) $)^{-1 / 2}$ and a NUV-appropriate Gaussian PSF having FWHM of 5.3" (3.5 pixels). In practice, the GALEX PSF varies subtlely from visit to visit and slightly as a function of position within the field of view (Morrissey et al. 2007). We further explored this issue, and our approach to dealing with it is described in the next section.

\subsection{Aperture and PSF Photometry}

Because of the expected variance in the PSF shape discussed above, we performed both aperture and PSF photometry on the entire set of detected sources (independently for each visit). Preliminary aperture photometry on NUV imaging (and FUV when available) was first performed with apertures of radius $5^{\prime \prime}$ and $10^{\prime \prime}$. After applying appropriate aperture corrections (taken from Morrissey et al. 2007), we found that the results from both aperture sizes matched well, fully consistent with most differences expected due to the presence of close neighbors more frequently falling in the larger aperture. However, in dense stellar fields, where crowding is a concern, we need to implement a different method for performing accurate photometry. In these regions, the need for PSF photometry becomes critical. The PSF-fitting method allows us to dissect significantly blended sources.

The IDL-based PSF-fitting routine we used was developed by T. Small (personal communication) as a robust deep field method for GALEX datasets. The source positions adopted for the PSF photometry were determined on the NUV imaging via DAOfind (as described in Sec. 3.1). Careful inspection showed the average PSF supplied by the GALEX project (www.galex.caltech.edu/researcher/techdoc-ch5.html\#2) was not an accurate enough representation of our sources for individual exposures, often not accounting for slight PSF ellipticity seen in individual visits, especially for data taken in the late part of the mission. The variation of the PSF across a single tile (at least inside a radius of $0.55^{\circ}$ ) was not as significant as intra-visit changes. For improved photometric accuracy we implemented an automated method for determining each observation's PSF. To perform this task, we first ran the PSF fitting code with the GALEX supplied mission-average PSF. We used the results of this first run to determine sources that are both bright and isolated enough to represent the PSF. A cut was made to exclude stars with nearby neighbors, and all other neighbors within 90" were masked. The 100 most significant of these stars were recentered to a reference star. The total flux for each star was normalized across this subset, maintaining the overall PSF shape. A new PSF was generated from a datacube of 100 such stars, taking the median for each pixel. The photometry was then recomputed with the new empirical PSF. As expected, we find that the custom PSF fitting better recovers the $5^{\prime \prime}$ aperture photometry than the first run (for isolated sources), and importantly deblends crowded sources.

\subsection{Photometric Completeness}

The exposure depth and source crowding both vary across our survey, making it important to estimate completeness limits for separate regions using artificial star insertion and

recovery techniques. Such work is now being conducted, but is beyond the scope of this 


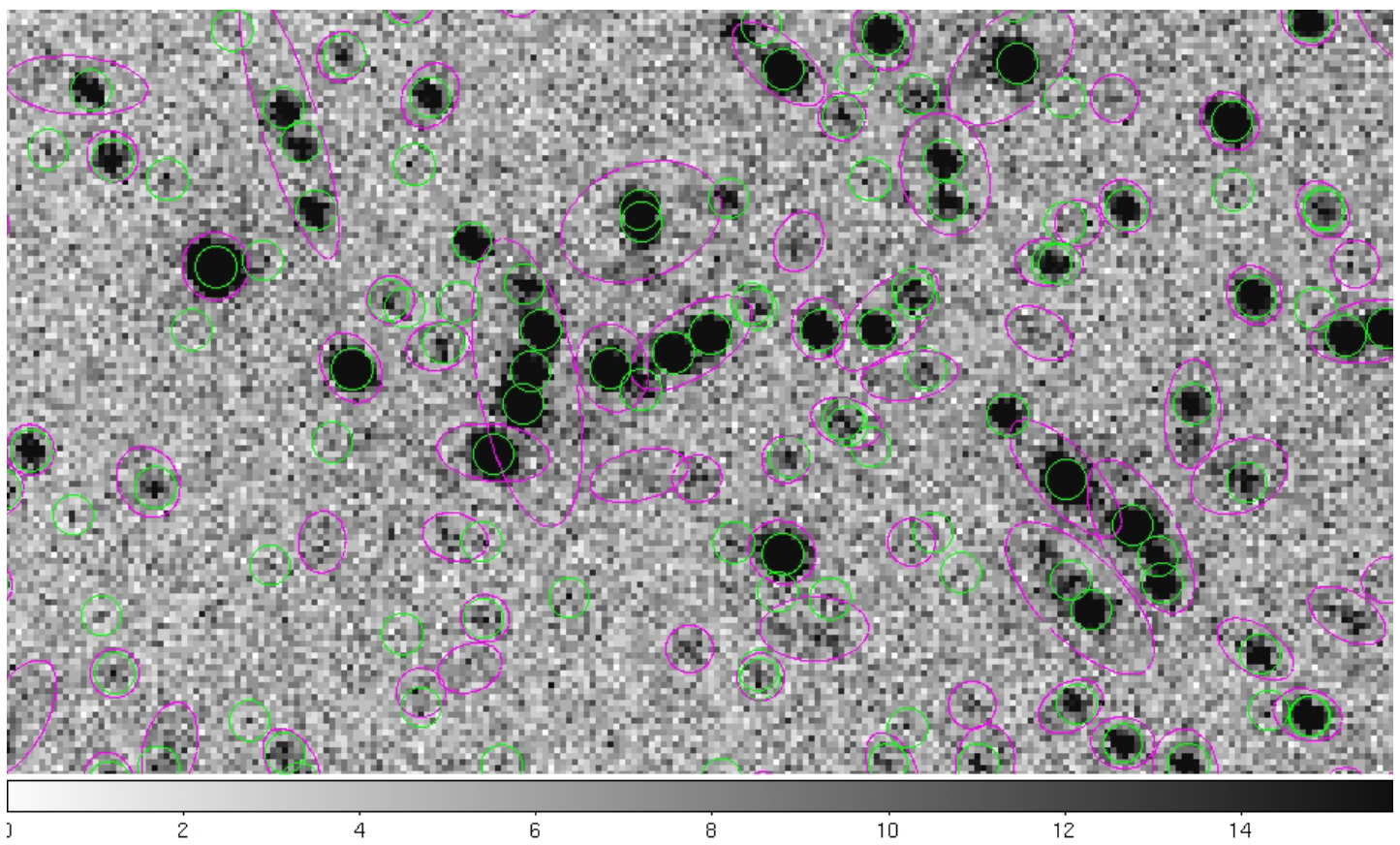

Figure 3: Example NUV count rate image with a comparison between GALEX-pipeline (purple) and our DAOfind (green) source detections for a tile in the LMC (visit exp. 1017s). In crowded fields, the GALEX pipeline fails to separate close and overlapping sources. Crowded regions of multiple sources are misidentified as elongated single detections by the pipeline. The DAOfind detections have not yet been pruned by significance, sharpness, or roundness at this pre-merge (see Sec. 4) stage.

short contribution. Standard Galex statistical estimates place the $5 \sigma$ point-source detection limit at NUV $\sim 22.7$ and FUV $\sim 22.6$ for MIS-depth (1500s) uncrowded observations. Within the crowded Magellanic Clouds, we will likely not reach such fiducial limits for single visit photometry (even for similar exposure times). For the present catalog, the visit level completeness is estimated to be NUV=19.5, FUV=19 AB mag at the shortest exposures $(\sim 100 \mathrm{~s})$. However, these short AIS exposures represent a small fraction of our survey (Fig 2 , top).

\section{Description of the Source Catalogs}

We have constructed comprehensive catalog of unique GALEX sources in the Large Magellanic Cloud. A similar product is currently being generated for the remaining survey area, including all visits described in Section 2.

After performing photometric measurements for sources detected independently in each GALEX visit, we had a large database containing multiple detections of astrophysically unique sources due to the overlap of adjacent tiles and also stemming from repeated visits. For the LMC visits analyzed in depth here (Fig. 4 and Sec. 2), this concatenated database had nearly 17 million detections. To produce a catalog of unique UV sources: (1) we first applied quality cuts on the concatenated source list, removing detections farther than $0.55^{\circ}$ from the field center or with DAOfind roundness/sharpness deviating from the median by greater than $\pm 2 \sigma$, together cutting the detection count to 11.3 million, (2) we then identified 
sources having repeated measurements within $2.5^{\prime \prime}$ separation, (3) for each set of coincident detections, the one having the lowest error was retained, except in comparatively rare cases of an equal error for which the source positioned closest to its field center was kept, (4) photometry measurements from multiple detections of each unique source were averaged, (5) finally a significance cut was applied to retain all unique sources having NUV error less than $0.5 \mathrm{mag}$. The final source catalog contains measured positions, image quality indicators (sharpness/roundness/crowding), PSF magnitudes and errors, plus reference to the individual detections prior to the catalog merge step.

Our output list of unique UV sources (drawing only from the non-AIS set of LMC visits) totals 5.8 million. Although this preliminary set of analyzed visits is not inclusive of all available data, it does contain the entire traditional extent of the LMC and can therefore be considered representative of our final LMC database. Plans for public release of this catalog are described in Section 7.

\section{The UV Source Content of the LMC Catalog}

Using the list of unique UV sources, we then generated maps of source surface density to illustrate the overall spatial distribution of NUV-detected point-like sources in the LMC. This was accomplished by counting the number of unique sources brighter than a threshold NUV magnitude falling into a grid of square sky tassels covering a $15^{\circ}$-wide field of view centered on the LMC. We adopted a tassel size of $0.04 \mathrm{deg}$ per side, corresponding to $35 \mathrm{pc}$ at the average distance of the LMC. Work is underway to generate improved maps incorporating color cuts and an adaptive-size smoothing kernel, but we present our results for the NUV sources here. Because the visits scattered across the galaxy are of varied exposure depth, it is not straightforward to produce a surface density map without incompleteness effects becoming apparent if the threshold magnitude is fainter than the limit associated with the shallowest visits. Therefore we generated source density maps adopting different choices of threshold magnitude. In the lower panel of Fig. 4 we show a section of the map resulting when a relatively bright threshold of NUV $=17.5$ ABmag is used. This map does not suffer from discontinuities due to exposure time differences, and highlights the small scale structures of star forming complexes. Such a map could be used to define star-forming complexes of UV-bright sources (e.g. Kang et al. 2009, Bianchi et al 2012b, Bianchi et al. 2013, this book). In the main panel of Fig. 4 we display the surface density map of sources with NUV $\leq 19.0$ ABmag. This view illustrates the overall extent of the LMC as traced by UV-bright sources, but the tile outlines of the deeper visits begin to become apparent. In the deeper map, we are able to trace the galaxy down to a source surface density of $0.09 \mathrm{UV}$-emitting stars $\operatorname{arcmin}^{-2}$ (430 stars kpc ${ }^{-2}$ ) which yields an apparent LMC diameter of approximately 10 degrees. The highest surface density observed for stars brighter NUV $=19.0 \mathrm{ABmag}$

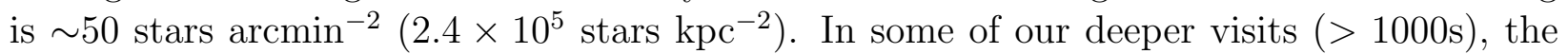
observed surface density of all detected sources approaches levels $10 \times$ greater than the values we quoted, demonstrating the need for PSF fitting photometry. We note that the observed source densities trace the intrinsic counts but are strongly modulated by extinction, to which UV fluxes are very sensitive (see also Bianchi 2011 and Bianchi et al., this book). In addition, they represent a lower limit to the actual number of hot stars, because the crowded cores 


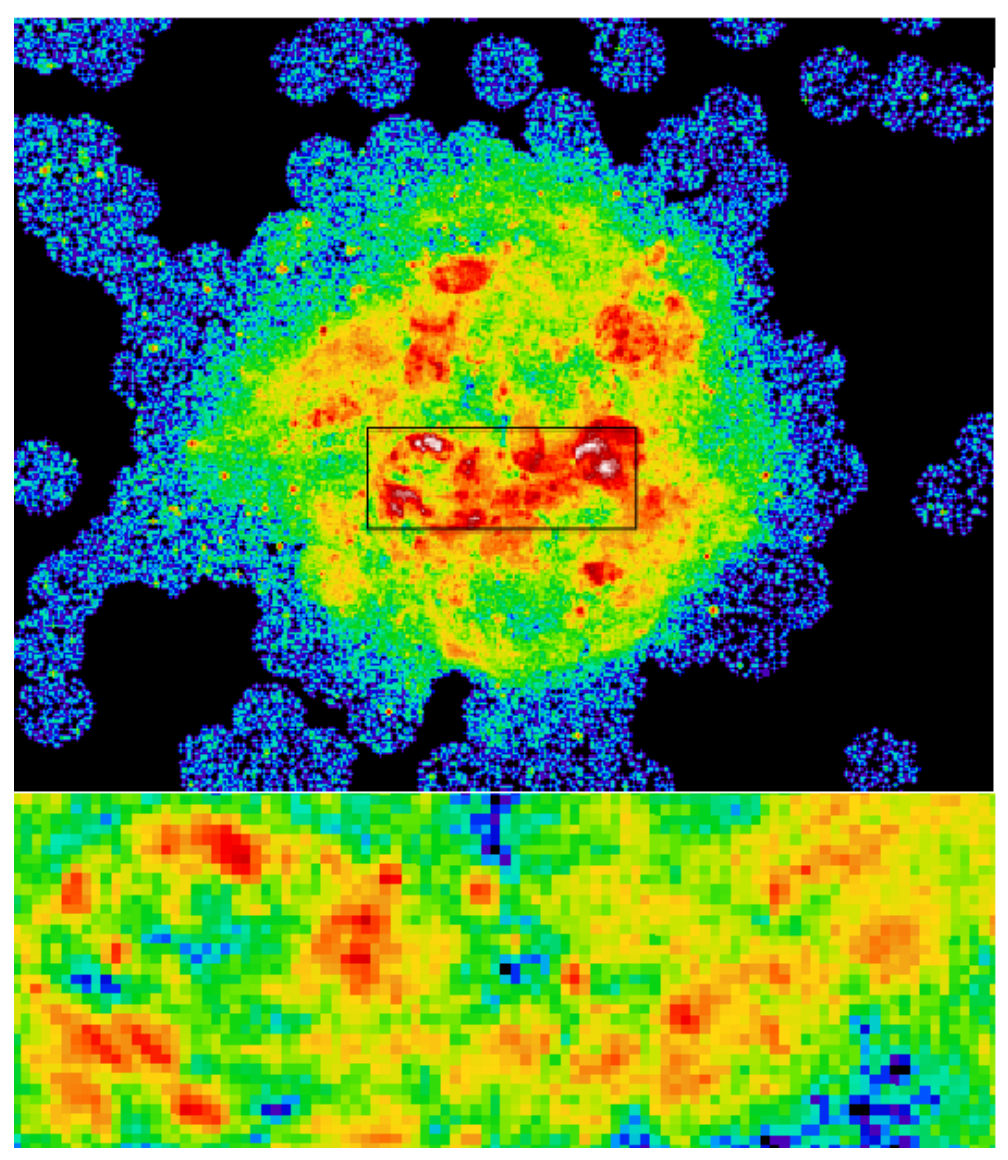

Figure 4: Surface density of NUV sources brighter than 19.0 mag in the LMC (main panel), evaluated over a $15^{\circ}$ wide field of view using only the non-AIS visits analyzed in this contribution. Some arc-like discontinuities are due to different relative completeness across tile boundaries with varied exposure time. The inset (boxed) shows a magnified view of a smaller area using a more restrictive cut (17.5 mag), helping to delineate small scale structures. Density is estimated across the galaxy counting sources in square tesselation bins of 0.04 deg extent per side. The intrinsic stellar density is modulated by local extinction that can severely reduce the counts. Levels depicted in these maps are discussed in the text. The detected extent of bright NUV point-sources (above the background surface density) is approximately equal to the HI extent of the galaxy at $\mathrm{N}(\mathrm{HI})=10^{20} \mathrm{~cm}^{-2}$. In this image $\mathrm{N}$ is up and $\mathrm{E}$ is left, slightly different from the orientation in Figs. 1 and 2 (which are on a different projection due to the wider area). Comparison is enabled by the coordinate grid shown in these earlier plots.

of star-forming regions and clusters contain many unresolved sources extended sources that are excluded from the point-source catalog, and will be treated seperately.

\section{Future Expansions of the Catalog and Sample Science Applications}

The GALEX UV imaging of the Magellanic System will support a wide range of science applications, including studies of the point source population (such as we have already started) but also various investigations based on unresolved UV features. We now briefly describe examples of such studies (e.g. stellar clusters, diffuse emission). We further note works which will be made possible by our future point source catalog expansion (beyond 
the LMC, multi-wavelength matching). Though not discussed here, it will be instructive to study the multi-wavelength morphology of the LMC and SMC, contrasted over the UV to IR (Miexner et al. 2006) and versus tracers of the gaseous interstellar medium (HI and CO).

\subsection{Stellar Clusters and Star-Forming Complexes}

Much attention has been focused recently on the properties of young-to-intermediate age star clusters in nearby galaxies. This comes from two coupled lines of inquiry: (1) in low mass clusters, incomplete sampling of the stellar initial mass function at high mass has implications for the estimation of cluster mass and age (even for the youngest clusters) due to stochastic fluctuations in predicted/ observed photometric properties and (2) the evolution (dissolution) of clusters at intermediate ages, hence the viability of dissolved clusters as a key driver of the field stellar population. We will soon undertake analysis of a sample of known young clusters throughout the Magellanic System, including the Clouds and Bridge. These objects are taken from the samples of Baumgardt et al. (2013) for the LMC, and Bica et al. (2008) over a wider area. For each cluster we have extracted a GALEX postage stamp image and will soon measure the integrated NUV and FUV (when available) magnitudes. Together with existing measurements at longer wavelengths, these will be used to characterize mass, extinction, and age of each cluster. In Figure 5, to illustrate an extreme cluster environment, we show the GALEX observations of the starburst complex 30 Dor, comparing NUV and optical imaging in the inset. In Figure 6 we display a large number of clusters with more typical masses in the LMC sample (NUV only). In Figure 6 it is apparent that the characteristic UV luminosity and morphology of the clusters varies as a function of both age and mass, while luminosity must also be modulated by local reddening.

\subsection{Diffuse UV Emission}

Inspection of the GALEX imaging for the Magellanic Clouds clearly reveals the presence of diffuse UV emission filling the space between resolved stars and clusters. Figure 5 shows the diffuse light in the vicinity of 30 Dor as an example. Much of this flux is thought to originate as scattered light, correlated roughly with the amount of dust in the interstellar medium and the UV continuum from nearby massive stars. In more distant galaxies similar diffuse emission is observed, but at the GALEX angular resolution it is challenging to exclude the possibility of intermediate mass main sequence stars as a significant contributor to the apparently diffuse light. The proximity of the MCs offers the opportunity to accurately decompose the observed UV emission into resolved and unresolved components, recovering all the point sources producing appreciable UV. This is one of the goals of our PSF fitting photometry. The ratio of the total flux of the point sources versus the (sky-subtracted) original image will be used as an indicator of the observed diffuse fraction, prior to applying an [estimated] correction for differential extinction between the stars and the diffuse medium.

\subsection{Recent Star Formation in the Magellanic Bridge}

GALEX provides a panoramic view of the MB, enabling a complete census of its hot stellar population. Our observations (Fig. 7) show that the Western MB has a significant component of recent star formation unrepresented by the OB association catalog of Bica \& Schmitt (1995). We assume contamination by white dwarfs (expected in the Clouds) is minimal within the MB, because there is no evidence for an old stellar population from 

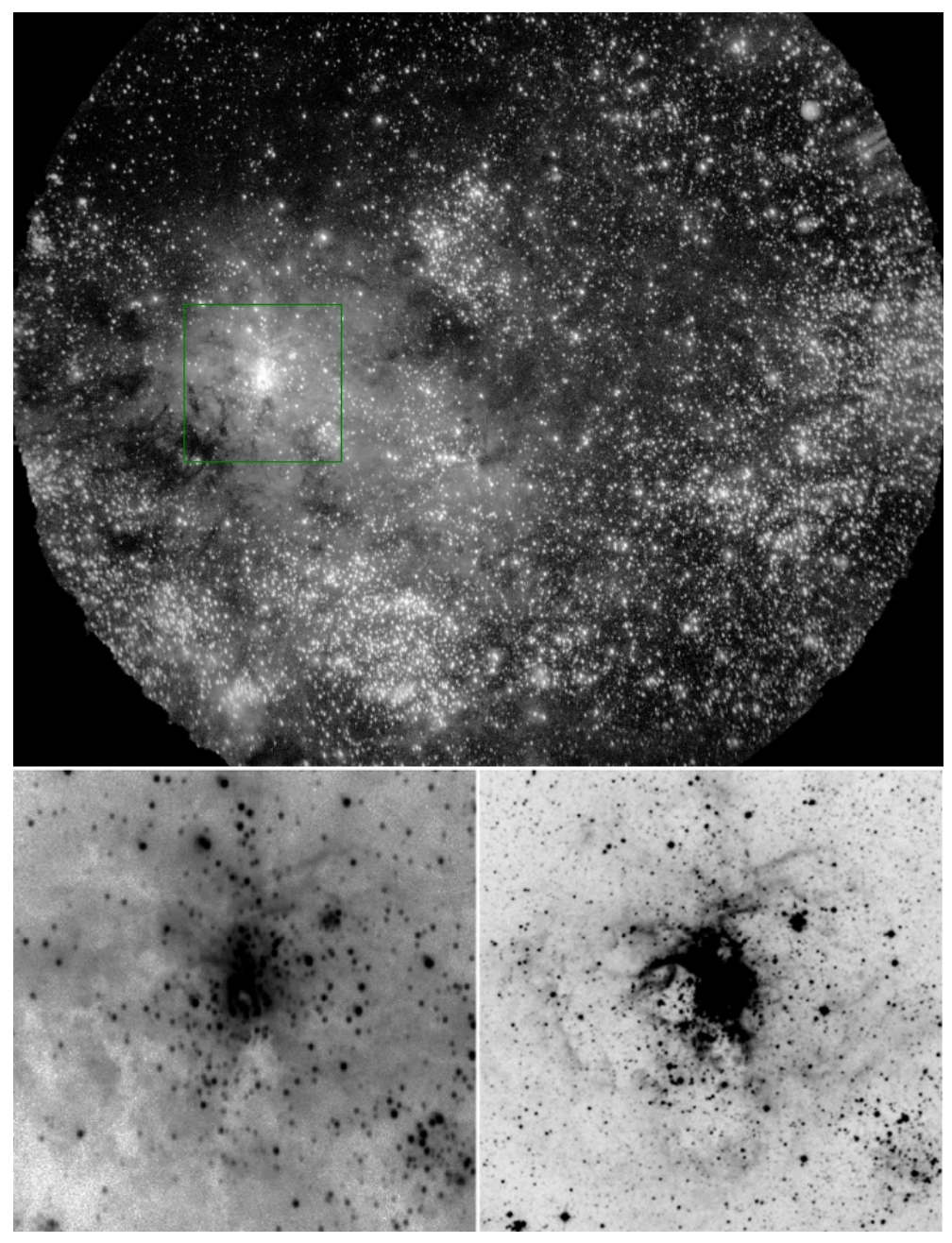

Figure 5: One GALEX tile containing 30 Doradus and its environment. A close up view in the UV (left) and optical (right) is provided in the inset, and the location of this $0.2^{\circ}(174 \mathrm{pc})$ box is marked in the wide-field image. The NUV image prominently displays the presence of dust, via bright regions of scattered light and also in absorption features (especially seen southeast of 30 Dor).

optical studies, even if this is untrue over our entire survey area. WD contamination will be explicitly checked from the UV-optical CMD after matching to ancillary imaging surveys. The top panel of Fig. 7 shows an $8^{\circ} \times 3.5^{\circ}$ portion of an FUV mosaic of the Bridge nearest the SMC. The missing area where the MB joins the SMC has been covered only in the NUV band. The importance of the FUV band for UV color selection of hot sources is apparent in the close-up view of one selected tile, shown at the bottom of Fig. 7 (with its location circled in the mosaic). In a forthcoming paper we will publish a catalog of UV-selected star-formation complexes and clusters in the MB, utilizing the type of unique source list described in Section 4 expanded to cover the entire GALEX MC survey region. 


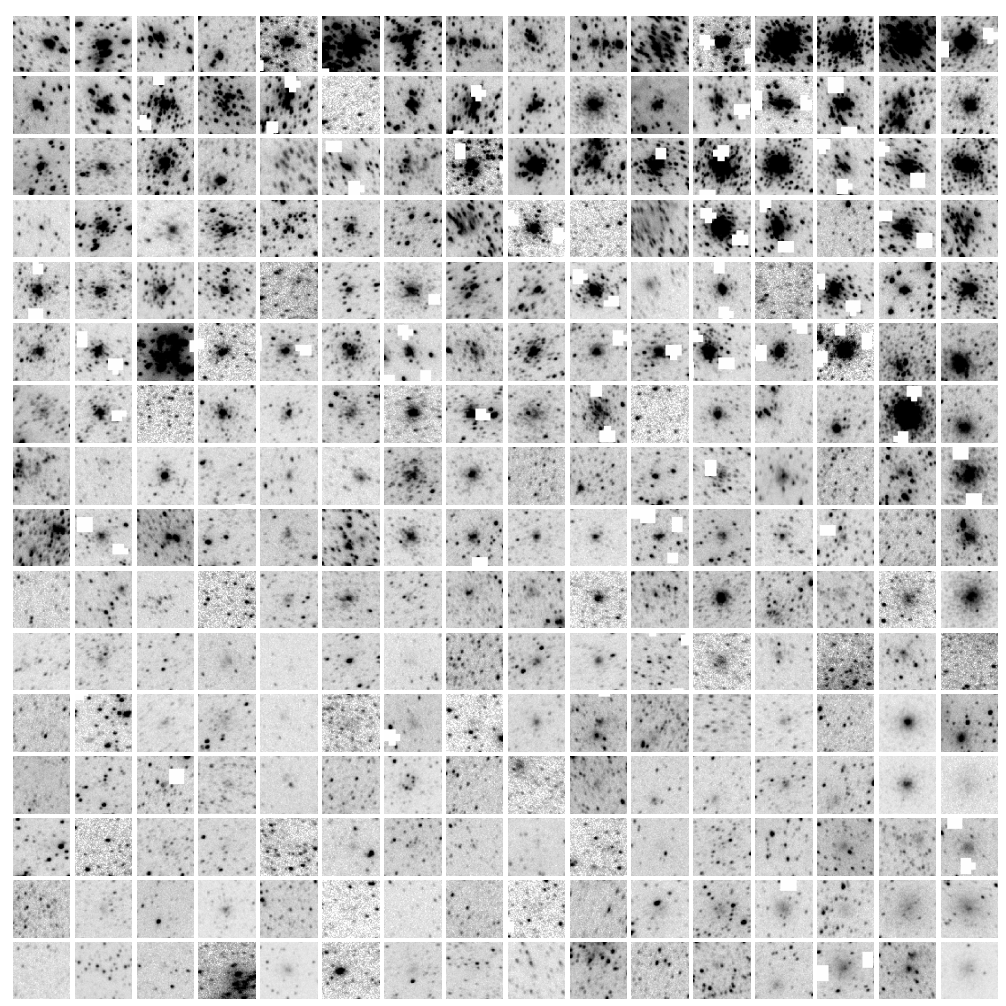

Figure 6: NUV images for 256 LMC clusters selected from Baumgardt et al. (2013). These are arranged such that the age increases downward and (within a row) the mass increases to the right. The median log (age) from Baumgardt et al. corresponding to the rows from top to bottom is 7.3, 7.4, 7.6, 7.8, 7.9, 8.0, 8.12, $8.2,8.3,8.4,8.6,8.73,8.97,9.05,9.13$, and 9.20 . The leftmost column typically corresponds to $\log \left(\mathrm{M}_{\odot}\right)$ about 3.7 whereas the value for rightmost column ranges from 4.3 to 5.2 depending on the observed cluster mass function within the age bin. The field of view shown for each cluster is $38 \mathrm{pc}$.

\subsection{Multi-wavelength Catalog Matching and Physical Parameters of the UV sources from UV-optical SED Fitting}

One of the aims of our future work is to estimate the physical parameters of the detected point-sources by spectral energy distribution (SED) analysis. This requires creation of matched catalogs linking the UV sources to their optical and near-IR counterparts. As a pilot study, we matched our UV data to the Magellanic Clouds Photometric Survey (Zaritsky et al. 2004), which covers an $8.5^{\circ} \times 7.5^{\circ}$ region of the LMC providing $U, B, V$, and $I$ magnitudes for most stars brighter than $\mathrm{V}=20$ Vegamag. The matching was done online using the CDS X-Match Service (http://cdsxmatch.u-strasbg.fr/xmatch), adopting a match radius of $2.5^{\prime \prime}$. Because the GALEX images have lower angular resolution, we tallied instances of multiple matches (more than one potential optical counterpart per UV source). UV sources with multiple optical matches inside the match radius must be treated with caution, since the UV flux may be the composite of multiple stars. These are about $20 \%$ of the present catalog. The GALEX and optical positions of matched sources are within $\lesssim 0.5^{\prime \prime}$ in the vast majority $(>90 \%)$ of cases, indicating consistency in the astrometry and robust matching between the two catalogs.

GALEX LMC sources with FUV and NUV photometry, and single optical counterparts 


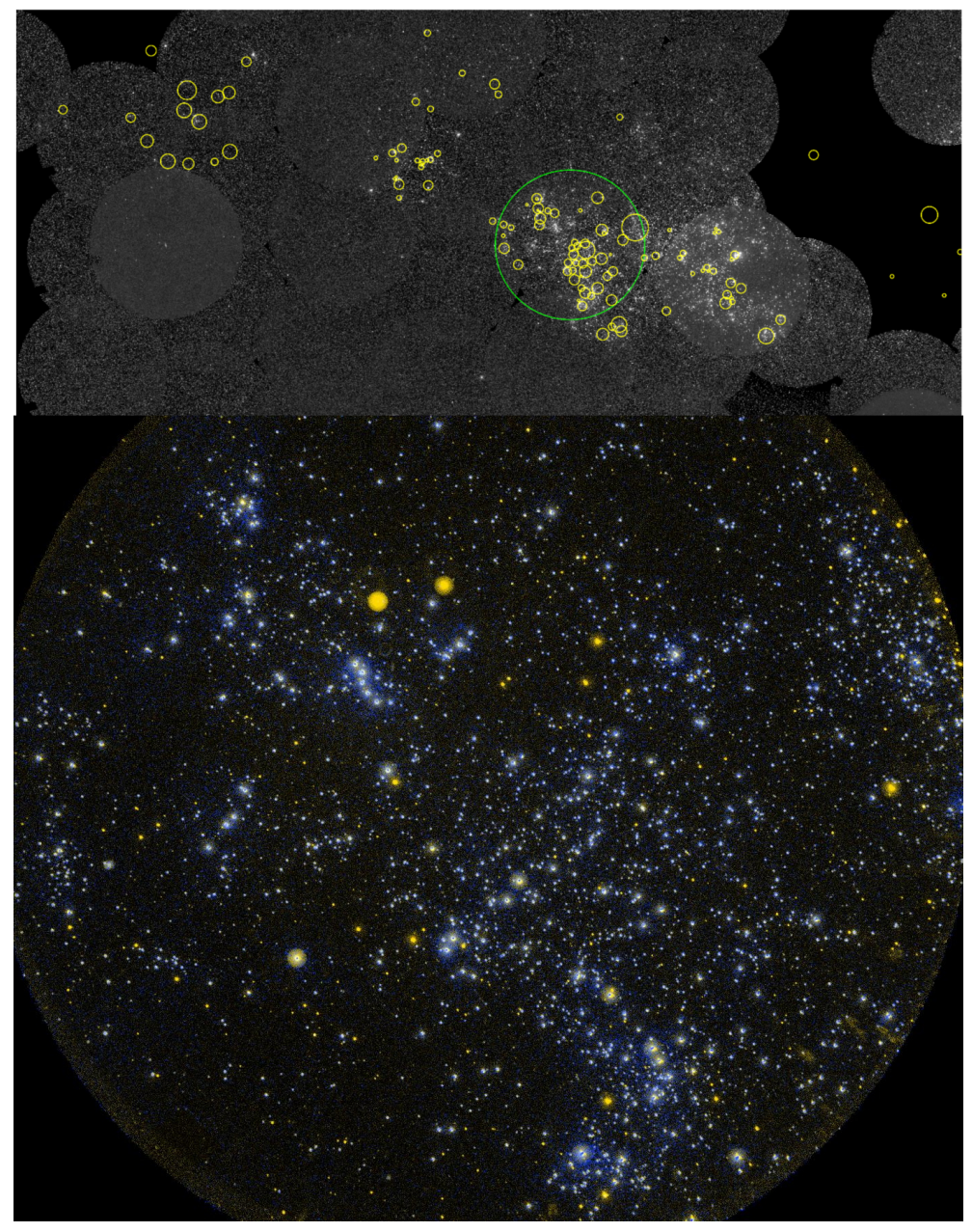

Figure 7: A ultraviolet view of the Magellanic Bridge from GALEX. The top panel shows a mosaic of FUV imaging for several visits and demonstrates the GALEX detection of recent star formation even outside the known OB associations (from Bica \& Schmitt 1995, and marked with yellow circles). The bottom panel presents a GALEX FUV, NUV (blue/yellow) color composite image for one selected tile. Its position in the mosaic is marked with a green circle, spanning about $1.1 \mathrm{kpc}$ in diameter.

(at $U B V I$ ), were analyzed with grids of stellar model atmospheres, reddened progressively assuming a variety of extinctions curves (see Bianchi et al. 2012a, b for details). The major parameters derived from SED fitting (through standard $\chi^{2}$ minimization), are the stellar effective temperature $T_{\text {eff }}$, and the extinction towards the source, $E_{B-V}$. The results depend on the assumed metallicity, and type of selective extinction $\left(\mathrm{A}_{\lambda} / E_{(B-V)}\right)$; the latter may significantly vary across different environments. The UV fluxes are particularly sensitive to this parameter (e.g., Bianchi 2007, 2011), and provide critical diagnostics for the hottest $T_{\text {eff }}$ 's. Because the distance to the stars is known, once $T_{\text {eff }}$ and $E_{B-V}$ are derived we can also obtain an estimate of the radius (and therefore $\mathrm{L}_{b o l}$ ) by scaling the best-fit model to the observed fluxes, accounting for extinction. A few examples of stellar SED and best-fit models are shown in Figure 8. We plan to expand this work to include all regions of both Clouds, even areas with only NUV coverage. 

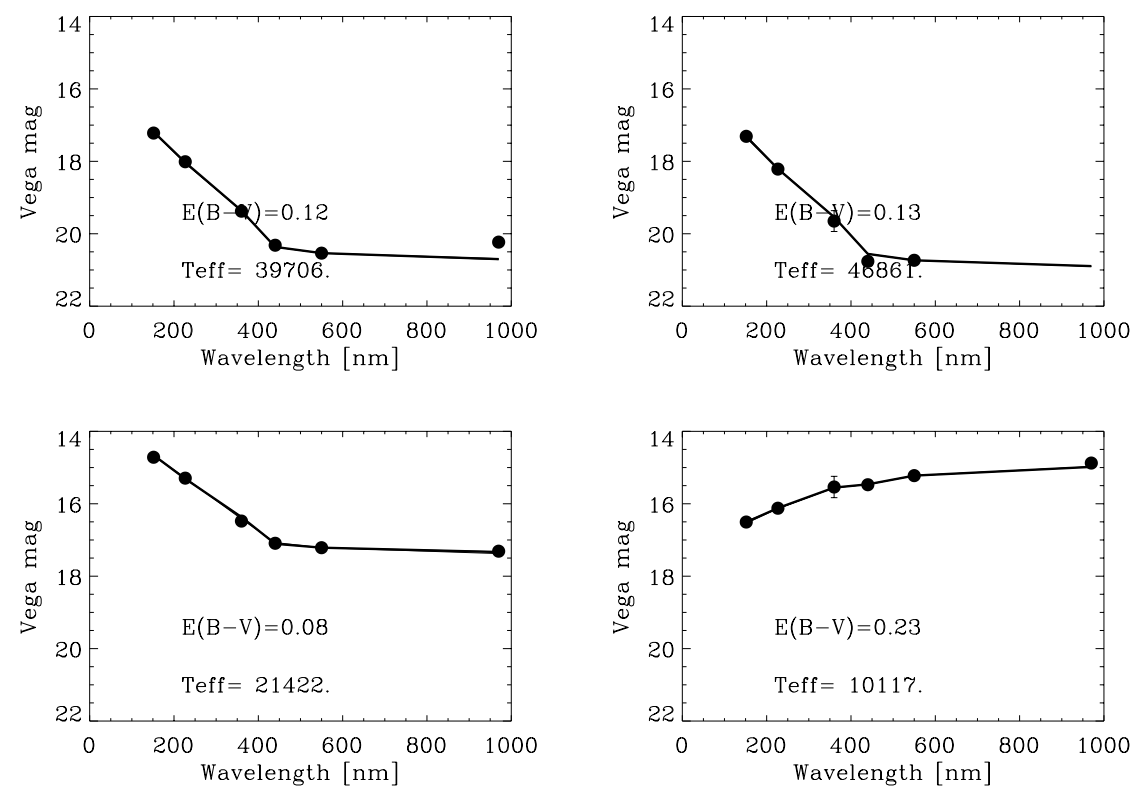

Figure 8: UV-optical photometry of stars in the GALEX catalog with existing optical data from the MCPS (Zaritsky et al. 2004). Dots are the photometric measurements, plotted at the $\lambda_{\text {eff }}$ of the respective bandpasses, the best-fit model magnitudes are connected by a line. Observational errors are smaller than the symbol size, with a few exceptions seen in the two righthand panels. The derived $T_{\text {eff }}$ and $E_{B-V}$ in each case are indicated. Most examples are chosen from the hottest stars. The SED fitting was done using the method of Bianchi et al. (2012a).

\section{Summary}

We have constructed a comprehensive catalog of unique GALEX UV sources in the Large Magellanic Cloud. A similar product is currently being generated for the remaining survey area, including all visits described in Section 2. The Magellanic Clouds will continue to remain an active area of interest in studies of low mass galaxy evolution, morphology and activity. Our survey provides a comprehensive UV assessment of the Magellanic Clouds, probing active resolved star formation regions in a low metallicity environment. The current contribution was intended to provide a first look at the LMC data, and highlight important forthcoming efforts based on the complete dataset (depicted in Figure 2). Our entire GALEX photometric catalog (including both LMC and SMC, plus surroundings) will be made available from the authors' website (dolomiti.pha.jhu.edu) and eventually from the MAST archive as a high-level science product (HLSP).

\section{Acknowledgements:}

We are grateful to the GALEX "Science Operation and Data Analysis Team" for their years of hard work and devotion which led to such a successful survey mission. This research has made use of the VizieR catalogue access tool, CDS, Strasbourg, France. In particular, the CDS X-Match service was very helpful. This research has made use of the NASA/IPAC Extragalactic Database (NED) which is operated by the Jet Propulsion Laboratory, Cal- 
ifornia Institute of Technology, under contract with the National Aeronautics and Space Administration.

\section{References}

Baumgardt, H., Parmentier, G. , Anders, P. \& Grebel, E. K., The star cluster formation history of the LMC, MNRAS, 430, 676-685, 2013.

Bianchi, L., Eframova, B., Hodge, P., et al., A Treasury Study of Star-forming Regions in the Local Group. I. HST Photometry of Young Populations in Six Dwarf Galaxies, AJ, 143, 74-98, 2012a.

Bianchi, L., Efremova, B., Hodge, P. \& Kang, Y., A Hubble Space Telescope Treasury Study of Star-forming Regions in the Local Group. II. Young Stellar Populations in M31, AJ, 144, 142-166, 2012b.

Bianchi, L. GALEX and Star Formation, AP\&SS, 335, 51-60, 2011.

Bianchi, L. The Ultraviolet sky surveys: filling the gap in our view of the Universe, AP\&SS, 320, 11-19, 2009.

Bianchi, L., Rodriguez-Merino, L., Viton, M., et al., Statistical Properties of the GALEXSDSS Matched Source Catalogs, and Classification of the UV Sources, ApJS, 173, 659-672, 2007.

Bica, E., Bonatto, C., Dutra, C. M. \& Santos, J. F. C., A general catalogue of extended objects in the Magellanic System, MNRAS, 389, 678-690, 2008.

Bica, E. L. D. \& Schmitt, H. R., A Revised and Extended Catalog of Magellanic System Clusters, Associations, and Emission Nebulae. I. Small Magellanic Cloud and Bridge, ApJS, 101, 41-85, 1995.

Brosch, N., Shara, M., MacKenty, J., Zurek, D. \& McLean, B., Far-Ultraviolet Imaging of the Field Star Population in the Large Magellanic Cloud with the Hubble Space Telescope, AJ, 117, 206-224, 1999.

Cheng, K.-P., Michalitsianos, A. G., Hintzen, P., Bohlin, R. C., O'Connell, R. W., Cornett, R. H., Roberts, M. S., Smith, A. M., Smith, E. P. \& Stecher, T. P., Astro-1 ultraviolet imaging of the 30 Doradus and SN 1987A fields with the Ultraviolet Imaging Telescope, ApJ, 395, L29-L32, 1992.

Cole, A. A., Tolstoy, E., Gallagher, III, J. S. \& Smecker-Hane, T. A., Spectroscopy of Red Giants in the Large Magellanic Cloud Bar: Abundances, Kinematics, and the AgeMetallicity Relation, AJ, 129, 1465-1482, 2005. 
Cornett, R. H., Hill, J. K., Bohlin, R. C., O'Connell, R. W., Roberts, M. S., Smith, A. M. \& Stecher, T. P., UIT: Ultraviolet observations of the small Magellanic cloud, ApJ, 430, L117-L120, 1994.

Cornett, R. H., Greason, M. R., Hill, J. K., Parker, J. W., Waller, W. H., Bohlin, R. C., Cheng, K.-P., Neff, S. G., O'Connell, R. W., Roberts, M. S., Smith, A. M. \& Stecher, T. P., UIT: Ultraviolet Observations of the Small Magellanic Cloud, AJ, 113, 1011-1021, 1997.

Hilditch, R.W., Howarth, I.D., Harries, T.J., Forty eclipsing binaries in the Small Magellanic Cloud: fundamental parameters and Cloud distance, MNRAS, 357, 304-324, 2005.

Hill, R. S., Cheng, K.-P., Bohlin, R. C., O'Connell, R. W., Roberts, M. S., Smith, A. M. \& Stecher, T. P., UIT and Optical Imagery of Large Magellanic Cloud Associations LH 52 and LH 53: Ages and Initial Mass Function Slopes, ApJ, 446, 622-636, 1995.

Kang, Y., Bianchi, L., Rey, S.-C., An Ultraviolet Study of Star-Forming Regions in M31, ApJ, 703, 614-627, 2009.

Martin, D. C., Fanson, J., Schiminovich, D.S., et al. The Galaxy Evolution Explorer: A Space Ultraviolet Survey Mission, ApJ, 619, L1-L6, 2005.

Meixner, M., Gordon, K. D., Indebetouw, R., et al., Spitzer Survey of the Large Magellanic Cloud: Surveying the Agents of a Galaxy's Evolution (SAGE). I. Overview and Initial Results, AJ, 132, 2268-2288, 2006.

Morrissey, P., Conrow, T., Barlow, T., et al. The Calibration and Data Products of GALEX, ApJS, 173, 682-697, 2007.

Olsen, K. A. G., Zaritsky, D., Blum, R. D., Boyer, M. L. \& Gordon, K. D., A Population of Accreted Small Magellanic Cloud Stars in the Large Magellanic Cloud, ApJ, 737, 29-37, 2011.

Page, T. \& Carruthers, G. R., Distribution of hot stars and hydrogen in the Large Magellanic Cloud, ApJ, 248, 906-924, 1981.

Parker, J. W., Hill, J. K., Cornett, R. H., Hollis, J., Zamkoff, E., Bohlin, R. C., O'Connell, R. W., Neff, S. G., Roberts, M. S., Smith, A. M. \& Stecher, T. P., Ultraviolet Imaging Telescope Observations of the Magellanic Clouds, AJ, 116, 180-208, 1998.

Parker, J. W., Zaritsky, D., Stecher, T. P., Harris, J. \& Massey, P., Ultraviolet and Optical Observations of OB Associations and Field Stars in the Southwest Region of the Large Magellanic Cloud, AJ, 121, 891-904, 2001.

Pietrzyński, G., et al., An eclipsing-binary distance to the Large Magellanic Cloud accurate to two per cent, Nature, 495, 76-79, 2013.

Pradhan, A. C., Murthy, J. \& Pathak, A., Observations of Far-ultraviolet Diffuse Emission from the Small Magellanic Cloud, ApJ, 743, 80-84, 2011. 
Putman, M. E., Gibson, B. K., Staveley-Smith, L., Banks, G., Barnes, D. G., Bhatal, R., Disney, M. J., Ekers, R. D., Freeman, K. C., Haynes, R. F., Henning, P., Jerjen, H., Kilborn, V., Koribalski, B., Knezek, P., Malin, D. F., Mould, J. R., Oosterloo, T. and Price, R. M., Ryder, S. D., Sadler, E. M., Stewart, I., Stootman, F., Vaile, R. A., Webster, R. L. \& Wright, A. E., Tidal disruption of the Magellanic Clouds by the Milky Way, Nature, 394, 752-754, 1998.

Smith, A. M., Cornett, R. H. \& Hill, R. S., Vacuum ultraviolet images of the Large Magellanic Cloud, ApJ, 320, 609-625, 1987.

Zaritsky, D., Harris, J., Thompson, I. B. \& Grebel, E. K., The Magellanic Clouds Photometric Survey: The Large Magellanic Cloud Stellar Catalog and Extinction Map, AJ, 128, 1606-1614, 2004. 